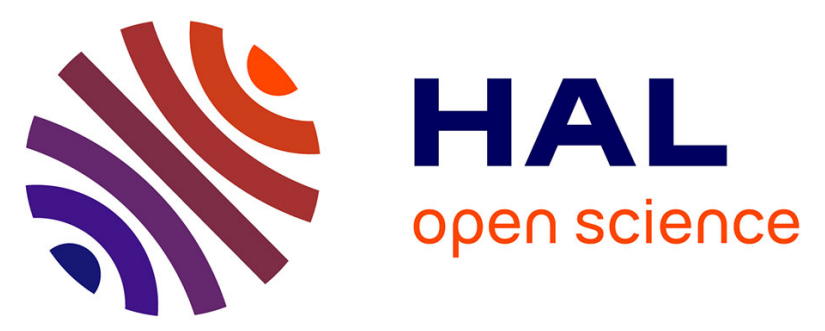

\title{
Expression patterns of LmAP2L1 and LmAP2L2 encoding two-APETALA2 domain proteins during somatic embryogenesis and germination of hybrid larch (Larix x marschlinsii).
}

Damien Guillaumot, Marie-Anne Lelu-Walter, Agnès Germot, Fanny

Meytraud, Louis Noël Gastinel, Catherine Riou-Khamlichi

\section{To cite this version:}

Damien Guillaumot, Marie-Anne Lelu-Walter, Agnès Germot, Fanny Meytraud, Louis Noël Gastinel, et al.. Expression patterns of LmAP2L1 and LmAP2L2 encoding two-APETALA2 domain proteins during somatic embryogenesis and germination of hybrid larch (Larix x marschlinsii).. Journal of Plant Physiology, 2008, 165 (9), pp.1003-1010. 10.1016/j.jplph.2007.08.009 . hal-01211910

\author{
HAL Id: hal-01211910 \\ https://hal.science/hal-01211910
}

Submitted on 31 May 2020

HAL is a multi-disciplinary open access archive for the deposit and dissemination of scientific research documents, whether they are published or not. The documents may come from teaching and research institutions in France or abroad, or from public or private research centers.
L'archive ouverte pluridisciplinaire HAL, est destinée au dépôt et à la diffusion de documents scientifiques de niveau recherche, publiés ou non, émanant des établissements d'enseignement et de recherche français ou étrangers, des laboratoires publics ou privés. 
Version définitive du manuscrit publié dans / Final version of the manuscript published in :

Journal of Plant Physiology 2008, 165(9), 1003-1010 ...

\section{SHORT COMMUNICATION}

\section{Expression patterns of $L m A P 2 L 1$ and $L m A P 2 L 2$ encoding two-APETALA2 domain proteins during somatic embryogenesis and germination of hybrid larch (Larix $x$ marschlinsii).}

Damien Guillaumota,1, Marie-Anne Lelu-Walterb, Agnès Germotc, Fanny Meytrauda, Louis Gastinela and Catherine Riou-Khamlichia*.

aGlycobiologie Végétale et Biotechnologie (EA3176), Université de Limoges, 123 avenue Albert Thomas, 87060 Limoges cedex, France

b INRA, UR588, Unité Amélioration, Génétique et Physiologie forestières, Avenue de la Pomme de Pin, BP 20619 Ardon, 45166 Olivet cedex, France.

c INRA, UMR 1061, Unité de Génétique Moléculaire Animale, GDR-CNRS 2590, Université de Limoges, 123 avenue Albert Thomas, 87060 Limoges cedex, France.

* Corresponding author.

E-mail address : catherine.riou@unilim.fr (C. Riou-Khamlichi)

Present address: Laboratory of Botany, Carnoy, Croix du Sud, 4-5 1348 Louvain la Neuve, Belgium

\section{SUMMARY}

Two APETALA2 domain transcription factors were firstly characterized in angiosperms and recently in several gymnoperms. These proteins are involved in several processes from flowering to embryogenesis in Arabidopsis thaliana and we extrapolated this result to hybrid larch (Larix x marschlinsii Coaz) resulting from a cross between European (Larix decidua) and Japanese (Larix kaempferi) larches. Somatic embryogenesis is a well described and controlled for this Pinaceae. We characterized two-AP2 domain genes named LmAP2L1 and $L m A P 2 L 2$. Phylogenetic analysis confirmed that LmAP2L1 and LmAP2L2 were orthologous to Norway spruce $P a A P 2 L 1$ and $P a A P 2 L 2$ and that L1 forms seemed to be specific to Pinaceae. RT-PCR analysis showed that larch APETALA2 were differentially expressed during late somatic embryogenesis and first steps of germination. Whereas LmAP2L2 was constitutively expressed during this process, $L m A P 2 L 1$ expression only appeared during late somatic embryogenesis when embryos were able to germinate. Furthermore, LmAP2L1 seemed to be the preferential expressed form during embryo germination. Thus, LmAP2L1 seemed to be a valuable molecular marker for hybrid larch late somatic embryogenesis and could play a role during post-embryonic development.

Key words: APETALA2; Hybrid Larch; Somatic embryogenesis

Abbreviations: aa, amino acid; AP2, APETALA2; EM, embryonal mass; L1, Larix $\mathrm{x}$ marschlinsii; Pa, Picea abies; Pt, Pinus thunbergii; RACE, rapid amplification of cDNA ends; RT-PCR, reverse transcriptase polymerase chain reaction; week, wk. 


\section{Introduction}

In gymnosperms, embryogenesis is divided into three distinct phases : proembryogeny, all stages before the elongation of the suspensor, early embryogeny before root and shoot meristem initiation and late embryogeny with primary meristem formation (von Aderkas et al., 1991; von Arnold et al., 2002). For gymnosperm embryogenesis, few molecular data are available due to the high complexity of this process especially during the two first phases, the absence of molecular databases and the lack of a conifer model such as Arabidopsis and poplar. Nevertheless, somatic embryogenesis which mimics zygotic embryogenesis is now well described for some gymnosperms such as spruce (Picea glauca (Moench) Voss), Douglas-fir (Pseudotsuga menziesii (Mirb.) Franco) and hybrid larch (Larix x marschlinsii Coaz) (von Aderkas et al. 1991; Lelu and Label, 1994; Gutmann et al., 1996; von Arnold et al., 2002). Of these three conifer models, somatic embryogenesis is fastest in hybrid larch, with embryos maturing in four weeks, by which time they have acquired the physiological capability to withstand desiccation and to initiate germination (von Aderkas et al. 2001, Mathieu et al., 2006).

To gain insight molecular markers of hybrid larch somatic embryogenesis, we focused our study on the APETALA2/ERF super family proteins, the two-AP2-domain proteins. These proteins are transcription factors involved in several developmental events from flowering to vegetative development in Arabidopsis, petunia and maize (Jofuku et al., 1994; Chuck et al., 1998; Riechmann and Meyerowitz, 1998; Maes et al., 1999). APETALA2 could also influence embryo development by controlling size and cell number (Jofuku et al., 1994, 2005; Ohto et al., 2005). APETALA2-Like genes were also characterized in Norway spruce (Picea abies (L.) Karst) and pine (Pinus thunbergii Parl.) (Vahala et al., 2001; Shigyo and Ito, 2004; Nilsson et al., 2007). We hypothesized that these proteins could also be characterized in hybrid larch, where they may play a role in somatic embryogenesis. We characterized two APETALA2-like cDNA from hybrid larch named LmAP2L1 and LmAP2L2. Using RT-PCR analyses, transcript expression patterns during somatic embryogenesis and germination of hybrid larch were examinated.

\section{Material and Methods}

Experiments were conducted with a highly embryogenic line of hybrid larch, Larix $\mathrm{x}$ marschlinsii (L. kaempferi (Lamb.) x L. decidua (Miller)) designated 69-18 obtained by secondary embryogenesis as previously described (Lelu et al., 1994 a,b). Embryonal mass (EM) was subcultured every 2 wks in darkness onto fresh proliferation medium which consisted of basal MSG medium supplemented with $0.01 \mathrm{M}$ glutamine, $9 \mu \mathrm{M} 2,4-\mathrm{D}, 2.3 \mu \mathrm{M}$ BA. Maturation was a step-wise process in which EM was first transferred for 1 wk to plant growth regulator-free MSG medium with $1 \%$ activated charcoal after which they were transferred for at least 4 wks onto MSG medium supplemented with ABA $60 \mu \mathrm{M}$ as previously described (von Aderkas et al., 2001). Four week-old cotyledonary somatic embryos were transferred onto plant growth regulator-free MSG medium for germination and plantlet development.

Total RNAs were extracted with a $70^{\circ} \mathrm{C}$ pre-heated CTAB extraction buffer as described by Chang et al. (1993). RNAs were quantified at 260 and $280 \mathrm{~nm}$ by spectrophotometry. Two $\mu \mathrm{g}$ of total RNAs extracted from EM and 4 week-old embryos after 14 or 25 days of germination were used to synthesize complementary DNA. The cDNA template obtained was amplified by PCR using AP2-domain-specific primers designed from Picea abies and Pinus thunbergii $P a A P 2 L 1$ and $L 2$ and PtAP2L1 and L2 nucleic acid sequences respectively (Vahala et al., 2001; Shigyo and Ito, 2004). 
For $L m A P 2 L 1$, primers used for 5' RACE were Sp1 : 5' TCATATGCCCTTGCAGCTTC3';Sp2 :5'CTGAATGATTCAGCTTGACGAAATGTAAG CC3', Sp3: 5'AACTTGGACTTCAGATCCCGGGCCATTGAC3' and for 3'RACE-PCR, Sp2:5'CTATATGAAAGTGAATTAGCCCTTACAGAGG3';Sp3:5'TGGGTTTGGCAACT GCCTACGAACAGGCCAG3'. For $L m A P 2 L 2$, primers used for 5' RACE-PCR were CAT 26: 5'ATG CGG GTT TCG CTG CTT C and CAT31: 5' CAGCGGAAAAAAATGGCGGGTCACGC and for 3' RACE-PCR were CAT27: 5'AGGATCGCGAAACCTTTAGC and CAT25 : 5'CTGCATAACCCGACCGGCGG. Accession numbers for LmAP2L1 and LmAP2L2 are DQ988340 and DQ988341 respectively.

Two $\mu \mathrm{g}$ of total RNAs extracted from the different tissues described below and from the different somatic embryogenesis stages were used to synthesize complementary DNA as detailed earlier. Primers were Lmcdc2sens

5'GAGCTAGCAAAGGATCCTCGTCTAATCA 3' and Lmcdc2 reverse: 5' CAGCTAATATACAGGTCAGGGTACAAAACC 3'; these primers amplified a fragment of $587 \mathrm{pb}$. Accession number for Lmcdc2 is DQ988343. For L1 and L2, we used a set of two primers CAT6 sens 5' CGTGGCGTGACATTCTACAG and CAT7 reverse 5' TTGGGGTGAGAATCCTGATG which allowed to visualize two fragments: a short fragment $(800 \mathrm{pb})$ corresponding to LmAP2L1 and a large fragment (1000 $\mathrm{pb}$ ) corresponding to $L m A P 2 L 2$ in the same PCR reaction (25 cycles and hybridization temperature $52^{\circ} \mathrm{C}$ ). Gel images were treated by the Image Quant TL (Amersham, UK) and the ratio: volume of control band (Lmcdc2) on volume of band corresponding to LmAP2L1 or LmAP2L2 was calculated. A ratio closed to 1 corresponds to a strong expression of transcripts.

The deduced amino acid sequences of two-AP2 genes were obtained from NCBI DNA databases. Sequences were aligned with the program CLUSTALW (1.83). Positions including gaps due to the alignment were removed. So, for phylogenetic analysis, 31 sequences and 133 positions were retained comprising the two AP2 domains and the linker. Maximum likelihood tree was obtained using the method implemented in the PROTML version 2.3 software (Adachi and Hasegawa, 1996). It was built by the quick-add Operational Taxonomic Units (OTUs) search, the Jones-Taylor-Thornton (JTT)-f model of amino acid substitution and retaining the 50-top ranking trees. Bootstrap proportions were calculated by the resampling estimated log-likelihood (RELL) method upon all top-ranking trees (Kishino et al., 1990). The best tree has a $\operatorname{lnL}$ of -1869.8 .

\section{Results and discussion}

Primers for cloning both forms of larch two-AP2-domain genes were designed from the sequences of Norway spruce and black pine (Vahala et al., 2001; Shigyo and Ito, 2004). The cDNA encoding LmAP2L1 was entirely characterized and encoded a putative protein of 529 aa containing the two-AP2 domain and a potential miR172 binding site (Figure 1A and 1C). Furthermore, L1AP2L1 sequence contained a conserved domain downstream the AP2 domain which function remains unknown (Figure 1B). This motif named $\mathrm{C}$ domain by Vahala et al. (2001) could be considered as a signature of Pinacea L1 forms. Indeed, the 10 aa motif (DPDLDLSLGNS) showed an aspartic acid in the third position which was only found in the gymnosperm L1 forms and monocotyledon plants like sweet potato, rice and hyacinthus whereas all the other plant sequences had an asparagine. In fact, LmAP2L1 putative amino acid sequence showed $86.59 \%$ identity with PtAP2L1 and PaAP2L1. In contrast, LmAP2L1 presented only $30.77 \%$ identity with $\mathrm{N}$ terminal truncated putative sequence encoded by LmAP2L2. Similar divergence was also observed for the L2 and L1 forms of pine and spruce species. Nevertheless, LmAP2L2 amino acid partial sequence (404 aa) showed a greater 
percentage of identity with the gymnosperm L2 forms (around $44 \%$ ) especially with PaAP2L2. This result linked to the phylogenetic analysis suggested that LmAP2L2 and PaAP2L2 are orthologs (Figure 2).

A 21 nucleotide micro-RNA, miRNA172, was completely complementary to Arabidopsis AP2 form which suggests that AP2L1 was its target (Chen, 2004). This was also shown for the regulation of glossy15 encoded a two-AP2 domain protein from maize (Lauter et al., 2005; Nilsson et al., 2007). L1 forms from Norway spruce, pine and hybrid larch had this site totally complementary and located at 3 ' end of cDNA. This motif was also described in primitive spermaphytes such as Ginkgo biloba, Cycas revoluta (Kim et al., 2006). Thus, a translation regulation by miRNA172 could down-modulate LmAP2L1 but further investigations should be necessary to show it and greatly interesting to enlarge the role of RNA interference in spermaphyte kingdom. By contrast, this site was really more divergent for the three AP2L2 forms suggesting that this protein was probably not regulated by translational regulation as we suppose it is the case for L1 forms.

Due to the high degree of amino acid conservation for AP2 domains and the fact that AP2 is a multigenic family, numerous nodes in the phylogenetic tree received low bootstrap supports weakening in some cases the evolutionary interpretation. Taking together, the 7 Pinaceae double AP2-domain proteins showed over the alignment portion more than $81 \%$ of identity. However, the three L1 forms were clearly orthologs, with a clade supported by bootstrap proportion (BP) of $100 \%$. The L2 forms looked more divergent (Figure 1 and Figure 2). Indeed, they did not form a monophyletic group in the tree. Only LmAP2L2 and PaAP2L2 could be recognized as orthologs with BP of 100\%. PtAP2L2 did not cluster with its counterparts. Two explanations could be proposed (i), the sequence of L2 form in pine is divergent due to a change in the selective pressure acting on the gene, or (ii) the sequence corresponds to a paralogous gene and the real ortholog of L2 form in pine had not been identified yet. A third form was recently described in Picea abies (Nilsson et al., 2007). PaAP2L3 is undoubtedly the sister group of Gnetum parvifolium AP2, with a bootstrap proportion of $98 \%$. Considering the phylogenetic link between gnetophytes and conifers (Hajibabaei et al., 2006) and the apparent single AP2 form present in G. parvifolium, Cycas revoluta and Gingko biloba (Shigyo et al., 2006), we hypothesized that L1 and L2 forms in Pinaceae could have arisen by lineage-specific duplications of an ancestral AP2L3-like gene.

We studied hybrid larch early and late somatic embryogenesis. Early somatic embryogenesis essentially corresponded to EM on proliferation medium, and late somatic embryogenesis corresponds to somatic embryo differentiation on activated charcoal and ABA media (von Aderkas et al., 1991). EM was a very proliferative tissue which contains a suspensor with very long cells and embryonal mass with actively dividing cells (Figure 3A). Detection of LmAP2L1 and LmAP2L2 transcripts was performed by RT-PCR analysis on 6918 EM. LmAP2L2 was highly expressed in EM (Figure 3B). Strong expression of PaAP2L2 was also observed in Norway spruce EM which is consistent with the fact the two forms are orthologous (Vahala et al., 2001). Moreover LmAP2L1 and PaAP2L1 transcripts were never detected in EM (Figure 3B; Vahala et al., 2001).

To gain insight into both patterns of gene expression, we induced somatic embryo differentiation from EM (Figure 3A). While LmAP2L2 expression decreased when EM was placed under activated charcoal condition, it rapidly increased after one week on ABA which corresponds to the beginning of the formation of globular shape somatic embryos (Figure 3AB). As described for $P a A P 2 L 2, L m A P 2 L 2$ expression remained high and relatively constant when differentiation occurred in 2 to 3 week-old embryos, which, by this time, had cotyledons and meristems (Figure 3A). As LmAP2L2 was then constitutively expressed, it was not a molecular marker of hybrid larch somatic embryogenesis. In contrast, LmAP2L1 expression started to be detected in 2 week-old somatic embryos which were not able to 
germinate (data not shown). This result suggests that LmAP2L1 could be a molecular marker of hybrid larch late somatic embryogenesis which did not seem to be the case for its ortholog PaAP2L1 (Vahala et al., 2001). LmAP2L1 expression corresponded to the moment when embryos started to store lipids and proteins and increase in mass (Lelu-Walter, personal communication). Indeed after 3 wks on ABA, mature embryos were able to germinate because they accumulated storage useful for further germination (Lelu and Label 1994, Gutmann et al., 1996; Label and Lelu, 2000). Our hypothesis is that, during late somatic embryogenesis, LmAP2L1 expression was linked to the capacity of embryos to germinate which was also a role suggested for Arabidopsis two-AP2 domain protein (Ohto et al., 2005). Nevertheless, even when embryos matured after 6 wks on ABA, both transcript forms were detected although LmAP2L2 expression level was always higher than LmAP2L1 (Figure 3B). As $L m A P 2 L 1$ remained expressed in very old embryos (12 week-old), it might have further roles that we should investigate.

Early vegetative growth was monitored on 4 week-old somatic embryos after 14 and 25 days of germination. After 25 days of germination, young conifers had green needles and well developed roots. Whereas $L m A P 2 L 1$ and $L m A P 2 L 2$ transcripts were strongly detected in somatic embryos germinating for 14 days (ratios closed to 1), both mRNA expression levels decreased after 25 days of germination. This was particularly observed for LmAP2L2 whose expression remained difficult to detect (Figure 3C). As AP2 proteins were known to be involved in organ formation, this result suggested that L1 and L2 forms could also act in further mechanisms. This was also consistent with previous work published by Jofuku et al. (2005) where APETALA2 was also detected in Arabidopsis leaves and stem.

\section{Conclusions}

So far, the two hybrid larch cDNAs encoding two-AP2 domain proteins constitute the first set of molecular data for this species. We showed that $L m A P 2 L 1$ is probably a good molecular marker of somatic late embryogenesis while LmAP2L2 was constitutively expressed during this fundamental process of plant cell life. Nevertheless, LmAP2L2 expression seemed to disappear during post-embryogenic development while LmAP2L1 remained quite highly expressed. Expression of $L m A P 2 L 1$ was clearly detected when embryos accumulate lipids and proteins and thus able to properly germinate. Furthermore, LmAP2L1 with its orthologs PaAP2L1 and PtAP2L1 constitute a gymnosperm specific sub-group. LmAP2L2 was also orthologous to PaAP2L2. As both forms were not regulated in the same manner at the transcriptional level, they are probably not redundant in function. Nevertheless, they could also be regulated at the translational level via microRNA172 especially LmAP2L1. It should be interesting to gain insight the role(s) of both forms by RNAinterference and transgenesis (Gleeson et al., 2005; Mathieu et al., 2006). Further investigations should be necessary to understand the roles of these two forms during embryogenesis and vegetative development.

\section{Acknowledgements}

We are grateful to Pr A. Oulmouden and L. Forestier for their help for cDNA sequencing. This work was supported by a Grant from the region Limousin (France).

\section{References}

Adachi J, Hasegawa M. Computer Science Monographs 1996; 28:1-150.

Chang S, Puryear J, Cairney J. Plant Mol. Biol. Rep. 1993; 11: 113-116.

Chuck G, Meeley RB, Hake S. Genes Dev. 1998; 12: 1145-1154. 
Chen X. Science 2004; 303: 2022-2025.

Gleeson D, Lelu-Walter M-A, Parkinson M. Mol. Breed. 2005; 15: 21-29.

Gutmann MP, von Aderkas P, Label P, Lelu M-A. J. Exp. Bot. 1996; 47: 1905-1917.

Hajibabaei M, Xia J, Drouin G. Mol. Phylogenet Evol. 2006; 40: 208-17.

Jofuku KD, den Boer BG, Van Montagu M, Okamuro JK. Plant Cell 1994; 6: 1211-25.

Jofuku KD, Omidyar PK, Gee Z, Okamuro, JK. Proc. Natl. Acad. Sci. USA. 2005; 102: 31173122.

Kim S, Soltis P, Wall K, Soltis D. Mol. Biol. Evo 2006; 23: 107-120.

Kishino H, Miyata T, Hasegawa M. J. Mol. Evol. 1990; 30: 151-60.

Label P., Lelu M-A. Physiol. Plant 2000; 109: 456-462.

Lauter N, Kampani A, Carlson S, Goebel M, Moose SP. Proc Natl Acad Sci USA. 2005; 102: 9412-9416.

Lelu M-A, Label P. Physiol. Plant. 1994; 92: 53-60.

Lelu M-A, Klimaszewska K, Charest P. Can. J. For. Res. 1994a; 24: 100-106.

Lelu M-A Bastien C, Klimaszewska K, Charest P. Plant Cell Tissue Organ Culture 1994b; 36 :

117-127.

Maes T, Van Montagu M, Gerats T. Dev Genet. 1999; 25: 199-208.

Mathieu M, Lelu-Walter M-A, Blervacq S, David H, Hawkins S, Neuterlings G. Plant Mol.

Biol. 2006; 61: 615-627.

Nilsson L, Carlsbecker A, Sundas-Larsson A, Vahala T. Planta 2007; 225: 589-602.

Ohto MA, Fischer RL, Goldberg RB, Nakamura K, Harada JJ. Proc Natl Acad Sci USA.

2005; 102: 3123-3128.

Riechmann JL, Meyerowitz EM. Biol. Chem. 1998; 379: 633-646.

Shigyo M, Ito M. Dev. Genes Evol. 2004; 214: 105-114.

Shigyo M, Hasebe M, Ito M. Gene 2006; 366: 256-265.

Vahala T, Oxelman B, von Arnold S. J. Exp. Bot. 2001; 358: 1111-1115

von Aderkas P, Bongas J, Klimaszewska K, Owens J. In Ahuja MR, editor. Woody Plant

Biotechnology, New York, Plenum Press, 1991, p139-155.

von Aderkas P, Lelu M-A, Label P. Plant Physiol. Biochem. 2001; 39: 1-8.

von Arnold S, Sabala I, Bozhkov PV, Dyachok J, Filonova L. Plant Cell Tiss. Org. Cult.

2002; 69: 233-249. 
Figure 1: Alignment of conserved regions of the two-AP2 domain proteins in conifer species, Larix $x$ marschlinsii (Lm), Picea abies (Pa) and Pinus thunbergii (Pt). A) Portion of the alignment used for the phylogenetic reconstruction. In bold the new sequences, LmAP2L1 and LmAP2AL2, described in this paper. R1 (yellow box) and R2 (blue box) correspond to sub-domains of the AP2 double domain (Kim et al., 2006), minus 2 amino acids at $\mathrm{N}$-terminal and 6 at $\mathrm{C}$-terminal, respectively. A 25 amino acid region also called linker region is located between $\mathrm{R} 1$ and $\mathrm{R} 2$ sub-domains. Stars point conserved residues and arrow heads, pertinent positions to discriminate L1, L2 and L3 forms. Are also shown for a given position, in green similar residues and in red non similar ones. B) A conserved domain is just found downstream to the AP2 double domain. Its consensus sequence is D P/Q D/N L D L S L G N/I S/G with PD and QN couples specific to L1 group and L2 group respectively. C) Putative binding site of miRNA172 in the cDNA of the 7 two-AP2 forms from Pinaceae. The nucleic acids that are identical to the complementary DNA of the miR172 are in bold. GenBank accession numbers for the different forms of AP2 genes are reported in material and methods section.

Figure 2: Maximum likelihood tree of the two-AP2 domain subfamily. Phylogenetic reconstruction was based on amino acid comparison of 31 double AP2-domain-containing genes selected from representative gymnosperms and angiosperms. It is rooted on the Aintegumenta (ANT) group, considering the existence of two monophyletic groups, AP2 and ANT, in the AP2 subfamily (Shigyo and Ito, 2004). GenBank accession numbers for the AP2 group are: CV006845 for Amborella trichopoda, AP2 NP_195410 and TOE1 NP_565674 for Arabidopsis thaliana, LIPLESS1 AAO52746 and LIPLESS2 AAO52747 for Antirrhinum majus, CrAP2L1 BAE48512 for Cycas revoluta, AP2 AAZ95247 for Dendrobium crumenatum, GbAP2L1 BAE48514 for Gingko biloba, GpAP2L1 BAE48516 for Gnetum parvifolium, HvAP2L1 AAL50205 for Hordeum vulgare, HAP2 AAD22495 for Hyacinthus orientalis, InAP2B BAD36744 for Ipomoea nil, LmAP2L1 DQ988340 and LmAP2L2 DQ988341 for Larix $\mathrm{x}$ marschlinsii, AHAP2 AAL57045 for Malus $x$ domestica, AP2D23 AAW78371 for Oryza sativa, PhAP2B AAD39440 for Petunia $x$ hybrida, PaAP2L1 AAG32658, PaAP2L2 AAG32659 and PaAP2L3 AAY79346 for Picea abies, PtAP2L1 BAD16603 and PtAP2L2 BAD16604 for Pinus thunbergii. Genbank accession numbers for the ANT group are: AINTEGUMENTA AAA91040, BABY BOOM AAM33803 and PLETHORA1 AAS86335 for Arabidopsis thaliana, BABY BOOM1 AAM33802 for Brassica napus, CrANTL1 BAE48513 for Cycas revoluta, GbANTL1 BAE48515 for Gingko biloba, (1) NP_915190 and (2) XP_474162 for Oryza sativa, PtANTL1 BAD16602 for Pinus thunbergii. The majority of these sequences except Amborella and Dendrobium were also described in previous works (Kim et al., 2006; Shigyo et al., 2006). Bootstrap values are indicated for branches supported by at least $50 \%$. The scale bar corresponds to 0.1 amino acid substitution per position. LmAP2L1 and LmAP2L2 of larch are in bold.

Figure 3: LmAP2L1 and LmAP2L2 gene expression patterns during somatic embryogenesis and germination of hybrid larch. RT-PCR was performed with primers allowing amplification of L1 and L2 forms in the same reaction. Upper band corresponds to LmAP2L2 (1000 bp) and the lower band to LmAP2L1 (800 bp). A) Pictures of EM and developing somatic embryos. Upper panel, from left to right: white EM (bar: $1 \mathrm{~mm}$ ), EM placed on activated charcoal for $1 \mathrm{wk}$ (AC, bar: $0.3 \mathrm{~mm}$ ), embryos on ABA for 1 to $2 \mathrm{wks}$ (bar: $0.5 \mathrm{~mm}$ ), for $3 \mathrm{wks}$ (bar: $0.5 \mathrm{~mm}$ ) and for 4 to $12 \mathrm{wks}$ (bar: $0.75 \mathrm{~mm}$ ). Pictures were taken under binocular microscope under visible light. Lower panel: 7 day-old EM observed 
under visible and fluorescence microscopy. DAPI staining was performed for observation of nuclei from EM and its suspensor. Bar: $100 \mu \mathrm{m}$. B) Expression of LmAP2L1 and LmAP2L2 gene transcripts during somatic embryogenesis of hybrid larch embryogenic line. EM; AC : EM placed on activated charcoal for one week; Lanes 1 to 7 correspond to embryos placed for 1 to 7 wks on ABA medium. Lane 8 corresponds to 12 wk-old embryos. C) Gene expression patterns of $L m A P 2 L 1$ and $L m A P 2 L 2$ during germination. Upper band corresponds to $L m A P 2 L 2$ and the lower band to $L m A P 2 L 1$. Lanes 1-2 correspond to 4 week-old somatic embryos after 25 and 14 days of germination, respectively. Lane 3 corresponds to 4 week-old somatic embryos non germinated. Normal condition of germination takes place under $16 \mathrm{~h}$ photoperiod. Numbers under pictures correspond to calculated ratios of LmAP2L2 (first line) and $L m A P 2 L 1$ gene expression levels compared to hybrid larch Lmcdc2 expression level, respectively. nd : not determined. Gene expression pattern was checked three times using three independent sets of plant material. 
AP 2L IP isus AP 2L IL arix AP $2 L$ IP ice AP $2 L 2 P$ i $c \in 2$ AP 2L 2L axix AP 2L 2P inus AP $2 \mathrm{~L} 3 \mathrm{P}$ ice

APQLLPim: AP2LIL arix sPQ LUP ice 2 APŹL\&Pice 2 AP2L2L arix APEL L APCLSPice

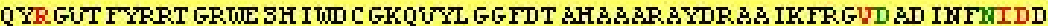
QYRGUT FYRRT GRWE SH IWD C GKQUYL GGFDT AHAABRAYDRAA IKFRGUD AD IMFIIDD QYRGUT FYRRT GRWE SH IWD CGKQUYL GGFDT AHABARAYDRAB IKFRGUD AD IMFIIDD QYRGUT FYRRT GRTE SH IWD C GKQUYL GGFDT AHAAAR AYDRAA IKFRGUD AD IMFTLTD QYRGUT FYRRT GRWE SH IWD C GKQUYL GGFDT AHAABRAYDRAA IKFRGUE AD INFTL SD QYRGUT FYRRT GRWE SH IWD C GKQUYL GGFDT AHAABRAYDRAA IKFRGQD AD IMFRL SD

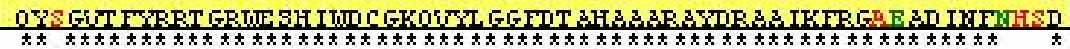

R.1

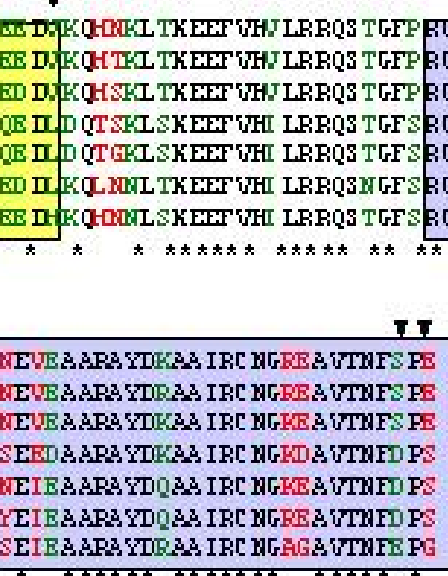
APELLPice 2 APQLZPFice 2 AP2L2L arix APCLLPing APQLSPice 2

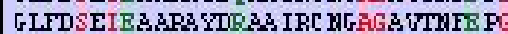

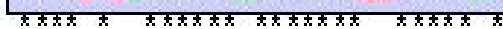

B

\begin{tabular}{|c|c|}
\hline $\mathrm{P}=\mathrm{AP} 2 \mathrm{~L} 1$ & DPDLDL SL GNS \\
\hline LmLP 2L 1 & DPDLDLSL GNS \\
\hline Pt AP 2L 1 & DPDLDL SLGNS \\
\hline Pt aP 2L 2 & DQKLDLSLGIS \\
\hline LmLP 2L 2 & DQRLDLSLGIS \\
\hline $\mathrm{P} Z \mathrm{AP} Z \mathrm{~L} 2$ & DQYLDLSLGIG \\
\hline$P$ בAP $2 L 3$ & DHRLL SL GMS \\
\hline
\end{tabular}

C

\begin{tabular}{|c|c|}
\hline LmLP 2L 1 & GT GC AGC AT CAT CAGGRTT CT \\
\hline P a AP 2L 1 & GT GC AGC AT CAT CPGGATTCT \\
\hline PtP A2L 1 & GT GC AGC AT CAT CAGGATTCT \\
\hline Pt AP 2L 2 & C AGC AGC AT CAT CAGGAT CT \\
\hline LmLPP 2L 2 & CT GCCGCAGTAT CAG-ATT CAT \\
\hline P בAP ZL 2 & GAT CCCCT GCAT CAGGATTCT \\
\hline$P=3 P 2 L 3$ & GT GC AGC AT CAT CDGGATTCT \\
\hline CDHA of miRMa & AT GCAGCATCATCABGATTCT \\
\hline miR172 & UACGUCGUAGUAGUUCUABGA \\
\hline
\end{tabular}

Figure 1 


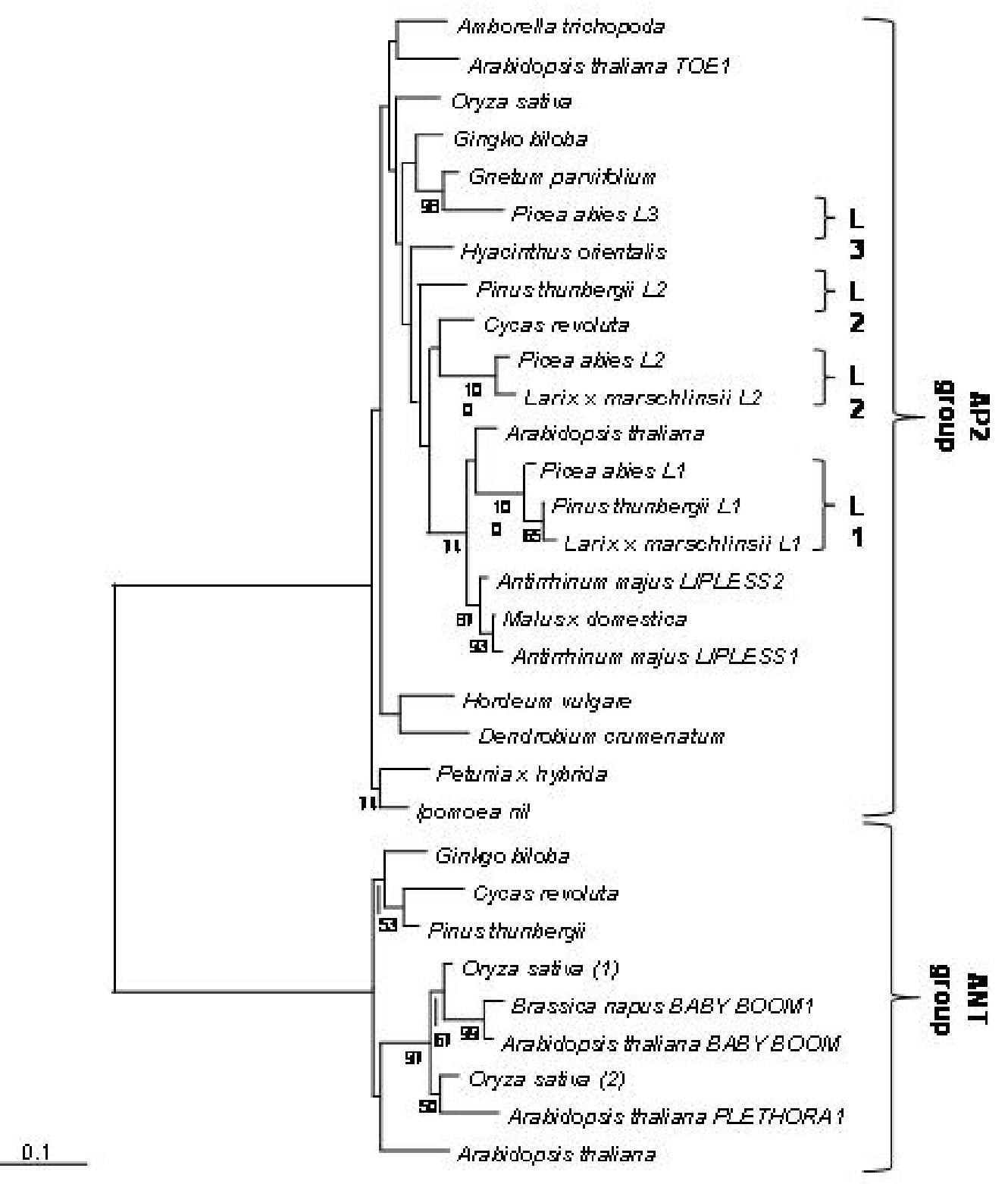

Figure 2 
$\mathbf{A}$
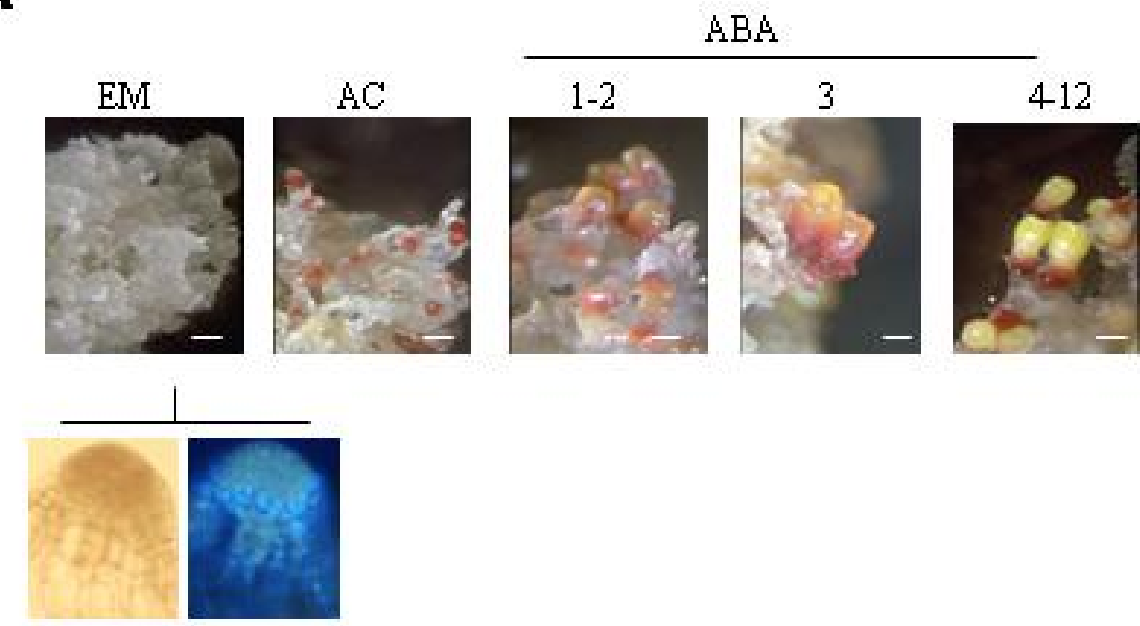

B

$\begin{array}{llllllllll}\mathrm{EM} & \mathrm{AC} & 1 & 2 & 3 & 4 & 5 & 6 & 7 & 8\end{array}$

\begin{tabular}{|c|c|c|c|c|c|c|c|c|c|c|}
\hline 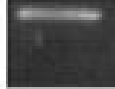 & & $=$ & 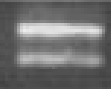 & $\underline{0}$ & $=$ & 5 & 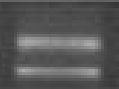 & $=$ & 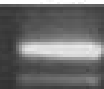 & $\begin{array}{l}L M A P 2 L 2 \\
I m A^{P 2 L I}\end{array}$ \\
\hline 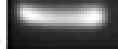 & 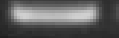 & 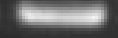 & 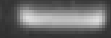 & 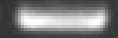 & 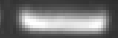 & 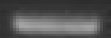 & 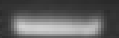 & 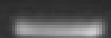 & & $\operatorname{Im} \in d 2$ \\
\hline & 8 & 2.00 & 2.4 & & 4.0 & 6.30 & 3.60 & 3.80 & 1.50 & \\
\hline $\mathrm{n}$ & nd & 46 & 4.30 & 5.70 & 4.80 & 11 & 4.50 & 3.40 & 8.70 & \\
\hline
\end{tabular}

C

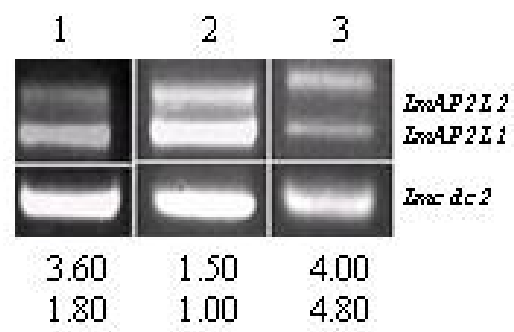

Figure 3 